\title{
Facteurs associés à la mauvaise qualité de la Gestion Active de la Troisième Phase de l'Accouchement dans les maternités du district de Dassa Zoumè- Glazoué au Bénin
}

\author{
J. SAIZONOU ${ }^{1 *}$, S. ADISSO ${ }^{2}$, D.V. AGUEH ${ }^{1}$, S. KEITA ${ }^{1}$ et M. MAKOUTODE ${ }^{1}$ \\ ${ }^{1}$ Institut Régional de Santé Publique, Ouidah, BENIN, 01 BP 918, Cotonou, Bénin; \\ ${ }^{2}$ Clinique Universitaire de Gynécologie Obstétrique du CNHU de Cotonou, Bénin ; Tel : +229 95154877 \\ *Auteur correspondant, E-mail : saizonoujacques@yahoo.fr; Tél : (229) $21341674 / 75$; 90923292 ; \\ Fax: (229) 21341672
}

\section{RESUME}

L'objectif était d'étudier les facteurs associés à la mise en œuvre de la Gestion Active de la Troisième Phase de l'Accouchement (GATPA) dans le district sanitaire de DAGLA au Bénin. Une étude analytique a été menée en juin 2011 portant sur les parturientes ayant accouché par voie basse dans sept maternités pratiquant la GATPA, les prestataires formés et le personnel de soutien. L'association entre la mauvaise qualité de cette intervention et les facteurs associés a été étudiée en utilisant le test de Khi carré de Pearson et la régression logistique pas à pas avec un seuil significatif fixé à 0.05 à l'aide de Epi Info 3.5.1. La mauvaise qualité de la GATPA a été de $63,2 \%$. Les facteurs associés étaient: la qualification des prestataires $(p=0.002)$, la supervision $(\mathrm{p}=0.034)$, les relations interpersonnelles $(\mathrm{p}<0.001)$, la satisfaction des parturientes $(\mathrm{p}=0.042)$. Dans le modèle final, la qualification $(\mathrm{p}=0.019)$ et les relations interpersonnelles $(\mathrm{p}<0.001)$ ont été les facteurs associés à la mauvaise qualité de la GATPA. Quelques dysfonctionnements ont été observés au niveau de l'organisation des services. Le renforcement des compétences techniques, la supervision régulière des prestataires et les relations soignants-soignés pourront contribuer à l'amélioration de la qualité de la GATPA dans le district sanitaire de DAGLA.

(C) 2012 International Formulae Group. All rights reserved.

Mots clés : Accouchement, gestion active, troisième phase, qualité.

\section{INTRODUCTION}

La gestion active de la troisième phase de l'accouchement (GATPA) est reconnue par la communauté internationale scientifique comme étant une intervention clinique à haut impact de prévention de l'hémorragie du postpartum (HPP) et de réduction de la mortalité maternelle (Prendiville et al., 2000; Burke, 2010). C'est pour cette raison qu'elle a été recommandée par l'Organisation Mondiale de la Santé (OMS) depuis 2002, pour tout accouchement par voie basse (Lawn et al., 2009; Abalos, 2009). En effet, l'hémorragie obstétricale est la première cause de mortalité maternelle dans le monde et contribue à plus de 33,9\% de décès maternel en Afrique (Kahn et al., 2006). L'hémorragie du post-partum en est la principale composante et chaque année 
dans les pays en voie de développement, 14 millions de femmes sont concernées (Bishop, 2008) ; elle survient à l'improviste chez toute femme, même sans aucun risque de pathologie, et peut entraîner le décès maternel en moins de deux heures si elle n'est pas prise en charge. La GATPA est par excellence la stratégie de prévention de l'HPP qui peut être évitée dans plus de $50 \%$ des cas, lorsqu'elle est pratiquée sur une femme immédiatement après la naissance de son bébé et avant l'expulsion du placenta (Lawn et al., 2009). Cependant, de nombreux services de santé dans les pays au sud du Sahara n'arrivent toujours pas à réaliser cette intervention ou bien elle est exécutée de façon insuffisante. Le nombre d'accouchements ne bénéficiant pas de GATPA est estimé à 1,4 millions, autant d'occasions pour éviter une HPP (Stanton et al., 2009). L'administration fiable de la GATPA, à l'instar d'autres interventions cliniques, dépend des fonctions essentielles du système de santé qui ont un faible niveau d'exécution dans les pays pauvres à forte mortalité (OMS, 2007 ; Cynthia et al., 2009). En effet, de nombreux obstacles sont observés pour intégrer la GATPA dans les soins courants de chaque accouchement. Une étude réalisée dans sept pays dont le Bénin, a montré une utilisation correcte de la GATPA dans seulement $0,5 \%$ à $32 \%$ des accouchements observés (Harvey et al., 2007 ; Stanton et al., 2009). La présente étude avait pour objectif d'étudier les facteurs associés à la mauvaise qualité de la GATPA dans le district sanitaire de Dassa-Zoumè-Glazoué (DAGLA) dans la perspective de surmonter les obstacles de sa mise en œuvre.

\section{Cadre d'étude}

Le district sanitaire de DAGLA est situé dans la région des Collines, à environ $255 \mathrm{~km}$ de Cotonou, la ville principale du Bénin. Elle couvre une superficie de 3475 $\mathrm{Km}^{2}$ pour une population estimée à 246650 habitants en 2010 (DDS-ZC du Bénin, 2010). Le climat est caractérisé par une saison pluvieuse de sept mois et une saison sèche de cinq mois. Les ethnies majoritaires sont les Idaatcha et Mahi. Les principales religions pratiquées sont le christianisme $(65,2 \%)$, la religion traditionnelle $(20,5 \%)$ et l'islam $(5,2 \%)$. L'économie est essentiellement agricole et commerciale. Les principales productions sont vivrières: maïs, haricot, arachide, manioc, igname, soja, riz et autres légumineuses. Sur le plan des infrastructures sanitaires, le district dispose d'un hôpital de zone, 30 centres de santé publics dont 23 disposent chacun d'une maternité. Dix sept centres de santé pratiquent la GATPA. Le district sanitaire de DAGLA a réalisé 5318 accouchements eutociques, 257 accouchements dystociques et 252 césariennes soit un total de 5827 accouchements en 2009. Il dispose d'un Collaboratif SONEGATPA qui est un cadre de concertation périodique pour l'amélioration continue de la qualité des Soins Obstétricaux et néonataux d'urgence et de la GATPA. Il est constitué des responsables des centres de santé et des maternités, et dispose en son sein des coachs internes et externes qui sont responsables de l'amélioration continue des prestations de soins.

\section{Type d'étude}

Il s'agit d'une étude transversale descriptive et analytique. La population de l'étude était constituée des parturientes admises dans les maternités dotées de personnel formé à la GATPA et ayant accouché par voie basse entre le 23 mai et le 16 juin 2011 ; le personnel de santé à savoir les sages femmes, infirmières et aides soignantes des maternités pratiquant la GATPA ainsi que des commis des postes de cession de médicaments.

La méthode d'échantillonnage était non aléatoire et la technique de choix était 
raisonnée. Le critère de sélection des maternités était basé sur le taux d'utilisation en accouchements ; ainsi ont été incluses dans l'étude les maternités les plus fréquentées et qui étaient: la maternité de l'Hôpital et six des 17 maternités périphériques pratiquant la GATPA et se trouvant à: Dassa-Zoumè, Kpingni, Paouignan, Glazoué, Magoumi et Sokponta. Toutes les parturientes admises dans ces maternités pour accouchement par voie basse ont été impliquées. Les sages femmes, infirmières et aides soignantes formées à la GATPA ainsi que les commis de cession de médicaments ont été également retenus.

\section{Variables de l'étude}

La GATPA est une intervention à trois composantes:

i) Administration d'un médicament utérotonique dans la minute qui suit la naissance du nouveau-né, après s'être assuré de l'absence d'un ou d'autres fœtus ; ii) Traction contrôlée sur le cordon ombilical tout en appliquant une contre-pression simultanée sur l'utérus à travers l'abdomen; et iii) Massage utérin à travers l'abdomen après l'expulsion du placenta, pour aider l'utérus à se rétracter afin de diminuer au maximum les saignements (Mathai et al., 2007). La définition de la GATPA adoptée par le Bénin est identique à celle recommandée par la Fédération Internationale des Gynécologues Obstétriciens (FIGO). Cette définition du Bénin comporte les trois composantes de la GATPA telles qu'énoncées par la FIGO et les neuf éléments tels décrits dans le Tableau 1 (Ministère Santé Publique du Bénin, 2009 ; PPHI, 2008).

\section{Variable dépendante}

Il s'agit de la qualité de la GATPA ; la qualité a été jugée mauvaise si un des neuf (9) critères cités dans le Tableau 1 n'était pas observé.

\section{Variables indépendantes}

Il s'agit des facteurs liés aux ressources humaines tels que la qualification, la formation en GATPA, la supervision, la motivation, les relations interpersonnelles; les facteurs liés à l'organisation et au fonctionnement des services tels que le planning des activités, l'utilisation du protocole clinique, la tenue des outils de gestion des données, la disponibilité et l'accessibilité aux utérotoniques, la conservation des utérotoniques; et les facteurs liés aux caractéristiques socioculturelles et aux antécédents gynéco-obstétricaux de la parturiente tels que l'âge, le statut matrimonial, le niveau d'instruction, la profession, la religion, l'ethnie, la gestité, la parité, les grossesses multiples, les interventions gynéco-obstétricales.

La collecte des données s'est déroulée du 23 mai au 16 juin 2011 dans les sept maternités ci-dessus citées. La collecte a consisté en : - l'observation des prestataires pratiquant la GATPA au cours des accouchements, des entretiens avec les parturientes, le personnel de soins, les commis ; - l'exploitation des fiches de stock des utérotoniques et autres documents utiles dans la pratique de la GATPA. La collecte des données a été réalisée par sept enquêtrices, deux sages femmes et cinq infirmières externes aux sites de l'étude, sous la supervision des auteurs de ce travail.

Le traitement des données a été à la fois manuel et informatisé. L'analyse des données a consisté au calcul des proportions et moyennes avec écart type pour les variables quantitatives; les données qualitatives ont fait l'objet d'analyse de contenu. Une association a été recherchée entre la variable dépendante avec chacune des variables indépendantes. Toutes les variables, dont le P-value qui était 
inférieur ou égal à 0.05 dans l'analyse bivariée, ont été mises dans un modèle global de régression logistique pas à pas descendante, pour étudier les facteurs de risque de la mauvaise qualité de la GATPA. Le test du Chi 2 du rapport de vraisemblance a été utilisé pour estimer la $\mathrm{P}$ - value globale des variables indicatrices du modèle. La valeur seuil de $\mathrm{P}$ a été fixée à 0.25 , afin de ne pas être trop restrictif et de ne pas exclure d'éventuels facteurs de confusion. La recherche d'interactions pertinentes a été réalisée. La mesure d'association donnée par la régression logistique est l'odds ratio (OR). Les intervalles de confiance ont été fixés à $95 \%$ et le logiciel Epi-Info version 3.5.1 a été utilisé pour réaliser ces analyses.

\section{RESULTATS}

\section{Description générale de l'échantillon}

L'étude a porté sur : - 174 parturientes qui ont accouché dans les sept maternités concernées. Leur âge moyen était de $26.5 \pm 06$ ans. Elles étaient pour la plupart mariées (82.8\%), non alphabétisées (51.7\%), femmes au foyer $(58.0 \%)$, multigestes $(44.2 \%)$, ou nullipares $(32.8 \%)$. Les femmes multipares représentaient $9.8 \%$.

-Vingt deux prestataires ont été inclus dans l'étude dont 13 étaient qualifiées (7 sages femmes et 6 infirmières). Parmi les sept commis des postes de cession de médicaments, trois étaient aides soignants et quatre étaient secrétaires administratifs adjoints.

\section{Mauvaise qualité de la GATPA}

Tous les éléments de la qualité de la GATPA ont été satisfaisants pour 64 accouchements sur 174. Par conséquent, la qualité de la GATPA était mauvaise dans $63.2 \%$ (110 / 174). Le délai d'administration de l'utérotonique $(75.3 \%)$, la contre pression sus-pubienne $(65.5 \%)$, le massage relayé par la parturiente $(83.3 \%)$ et le contrôle du tonus $(51.1 \%)$ étaient des facteurs de faible qualité de la GATPA (Tableau 2).

\section{Facteurs liés à l'organisation et au fonctionnement des services}

L'ocytocine a été disponible dans toutes les maternités de la zone d'étude et aucune rupture de stock n'a été observée. Les consommations moyennes mensuelles n'étaient pas connues des gestionnaires de médicaments; ce qui s'était traduit par l'observance de la date de péremption critique (moins de trois semaines) des ocytocines dans les centres de santé de Sokponta et de Dassa. Un mécanisme a été mis en place dans les sept maternités de l'étude pour rendre immédiatement accessible l'ocytocine; ce mécanisme est basé sur la conservation dans les salles d'accouchement de ce produit dans des porte-vaccins avec des accumulateurs congelés. Les registres d'accouchements ont été correctement remplis dans $80.5 \%$ des centres de santé. Le REMECAR (Registre de Médicaments, Carnet, Acte et Recettes) était bien tenu dans les sept formations sanitaires. La continuité des soins, selon les critères retenus, a été observée pour $62.6 \%$ des accouchements. Le port des équipements de protection individuelle (Bavettes, bottes, lunettes, bonnets et tabliers) et le lavage des mains avant et après les soins ont été observés dans $47.1 \%$ des accouchements. Le protocole clinique des services de santé maternelle était disponible dans les sept formations sanitaires. La conservation de l'ocytocine et les éléments de la chaîne de froid étaient respectés dans les maternités à l'exception de celle de Glazoué où elle était conservée à la température ambiante. Les températures mensuelles enregistrées ont varié de 3.16 à $4.20{ }^{\circ} \mathrm{C}$. 
Facteurs liés aux ressources humaines associés à la mauvaise qualité de la GATPA dans le district sanitaire de DAGLA

Des quatre facteurs liés aux ressources humaines, trois ont été statistiquement associés à la qualité de la GATPA (Tableau 3); il s'agit de la qualification des prestataires $(\mathrm{p}=0.002)$, la supervision $(\mathrm{p}=0.034)$ et les relations interpersonnelles $(\mathrm{p}<0.001)$.

Facteurs liés aux caractéristiques socioculturelles et aux antécédents gynécoobstétricaux associés à la mauvaise qualité de la GATPA dans le district de DAGLA

Le Tableau 4 montre que, des huit facteurs liés aux caractéristiques socioculturelles, aux antécédents gynécoobstétricaux des parturientes, un était associé à la mauvaise qualité de la GATPA ; il s'agit de la satisfaction de la parturiente $(\mathrm{p}<0.042)$.

\section{Analyse multi-variée des facteurs associés à la mauvaise qualité de la GATPA}

L'ensemble des variables indépendantes, dont le $\mathrm{P}$ - Value, est inférieur ou égal à 0.05 et a été mis dans un modèle global de régression logistique; il s'agit de la qualification des prestataires, la supervision, des relations interpersonnelles et la satisfaction des parturientes. Le modèle final a retenu la qualification des prestataires et les relations interpersonnelles comme étant des facteurs qui influençaient la qualité de la GATPA dans le district sanitaire de DAGLA (Tableau 5).

La mauvaise qualité de la GATPA est quatre fois plus observée quand elle est pratiquée par un personnel non qualifié et formé, et cinq fois plus observée en cas d'une mauvaise communication prestataire-patient.

Tableau 1 : Comparaison de la définition de la GATPA selon le Bénin et selon la FIGO.

\begin{tabular}{|c|c|c|}
\hline Composantes & Définition du Bénin & Définition FIGO \\
\hline \multirow[b]{4}{*}{ Utérotonique } & 1. Type : Ocytocine & 1. Type : Ocytocine \\
\hline & 2. Dose : $10 \mathrm{UI}$ & 2. Dose : $10 \mathrm{UI}$ \\
\hline & 3. Voie d'administration : IM & 3. Voie d'administration : IM \\
\hline & $\begin{array}{l}\text { 4. Délai d'administration : dans la } \\
\text { minute qui suit la sortie de l'enfant } \\
\text { (Précautions : palper l'utérus pour } \\
\text { écarter l'éventualité d'un } \\
\text { autre/d'autres bébé(s) avant } \\
\text { l'administration d'un utérotonique) }\end{array}$ & $\begin{array}{l}\text { 4. Délai d'administration : dans la } \\
\text { minute qui suit la sortie de l'enfant } \\
\text { (Précautions : palper l'utérus pour } \\
\text { écarter l'éventualité d'un } \\
\text { autre/d'autres bébé(s) avant } \\
\text { l'administration d'un utérotonique) }\end{array}$ \\
\hline \multirow[b]{2}{*}{ Traction contrôlée } & 5. Traction contrôlée sur le cordon & 5. Traction contrôlée sur le cordon \\
\hline & $\begin{array}{l}\text { 6. Avec une contre-pression sus } \\
\text { pubienne }\end{array}$ & $\begin{array}{l}\text { 6. Avec une contre-pression sus } \\
\text { pubienne }\end{array}$ \\
\hline \multirow{3}{*}{ Massage } & $\begin{array}{l}\text { 7. Massage utérin trans- abdominal } \\
\text { par l'accoucheur après la } \\
\text { délivrance }\end{array}$ & $\begin{array}{l}\text { 7. Massage utérin trans-abdominal } \\
\text { par l'accoucheur après la délivrance }\end{array}$ \\
\hline & 8. Massage relayé par la femme & \\
\hline & $\begin{array}{l}\text { 9. Contrôler le tonus utérin toutes les } \\
15 \text { minutes pendant } 2 \text { heures }\end{array}$ & $\begin{array}{l}\text { 8. Contrôler le tonus utérin toutes } \\
\text { les } 15 \text { minutes pendant } 2 \text { heures }\end{array}$ \\
\hline
\end{tabular}


Tableau 2 : Répartition des accouchements selon le respect des neufs critères de la GATPA.

\begin{tabular}{lcc}
\hline Critères de la qualité de la GATPA & Nombre & Pourcentage \\
\hline Type d'utérotonique & 174 & 100 \\
Dose d'utérotonique & 174 & 100 \\
Voie d'administration d' utérotonique & 174 & 100 \\
Délai d'administration d'utérotonique & 131 & 75.3 \\
Traction contrôlée sur le cordon & 174 & 100 \\
Contre-pression sus pubienne & 114 & 65.5 \\
Massage utérin par l'accoucheur & 173 & 99.4 \\
Massage relayé par la parturiente & 145 & 83.3 \\
Contrôle du tonus toutes les 15 minutes & 89 & 51.1 \\
\hline
\end{tabular}

Tableau 3: Association entre le niveau de qualité de la GATPA et les facteurs liés aux ressources humaines dans le district sanitaire de DAGLA.

\begin{tabular}{lccc}
\hline Variables & $\begin{array}{c}\text { Mauvaise qualité GATPA } \\
(\mathbf{n}=\mathbf{1 1 0})\end{array}$ & $\begin{array}{c}\text { Bonne qualité GATPA } \\
(\mathbf{n}=\mathbf{6 4})\end{array}$ & P-value \\
\hline Qualification & 25.5 & 06.3 & 0.002 \\
Non qualifié & 74.5 & 93.7 & \\
Qualifié & & & 0.034 \\
\hline Supervision & 52.3 & 40.6 & \\
Non supervisée & 47.7 & 59.4 & \\
Supervisée & & & 0.405 \\
\hline Motivation & 15.5 & 10.9 & \\
Non motivée & 84.5 & 89.1 & \\
Motivée & & & \\
\hline Relations & & & \\
interpersonnelles & 38.2 & 09.4 & \\
Mauvaises & 61.8 & 90.6 & \\
Bonnes & & & \\
\hline
\end{tabular}


Tableau 4: Association entre la mauvaise qualité de la GATPA et les facteurs liés aux caractéristiques socioculturelles, antécédents gynéco-obstétricaux dans le district sanitaire de DAGLA.

\begin{tabular}{|c|c|c|c|}
\hline Variables & $\begin{array}{l}\text { Mauvaise qualité GATPA } \\
\qquad(\mathrm{n}=\mathbf{1 1 0})\end{array}$ & $\begin{array}{l}\text { Bonne qualité GATPA } \\
(n=64)\end{array}$ & P-value \\
\hline Tranche d'âge (ans) & & & 0.239 \\
\hline$\leq 24$ & 41.8 & 32.8 & \\
\hline$>24$ & 58.2 & 67.2 & \\
\hline Niveau d'instruction & & & 0.101 \\
\hline Non scolarisée & 62.7 & 50.0 & \\
\hline Scolarisée & 37.3 & 50.0 & \\
\hline Gestité & & & 0.830 \\
\hline$\leq 3$ & 56.4 & 54.7 & \\
\hline$>3$ & 43.6 & 45.3 & \\
\hline Parité & & & 0.785 \\
\hline$>3$ & 28.2 & 26.6 & \\
\hline$\leq 3$ & 71.8 & 73.4 & \\
\hline $\begin{array}{l}\text { Utilisation } \\
\text { d'utérotonique pour } \\
\text { autres indications }\end{array}$ & & & 0.733 \\
\hline Non & 77.3 & 75.0 & \\
\hline Oui & 22.7 & 25.0 & \\
\hline $\begin{array}{l}\text { Interventions gynéco- } \\
\text { obstétricales }\end{array}$ & & & 0.648 \\
\hline Oui & 20.0 & 17.2 & \\
\hline Non & 80.0 & 82.8 & \\
\hline Grossesses multiples & & & 0.630 \\
\hline Non & 10.0 & 07.8 & \\
\hline Oui & 90.0 & 92.2 & \\
\hline Satisfaction & & & 0.042 \\
\hline Non & 25.5 & 12.5 & \\
\hline Oui & 74.5 & 87.5 & \\
\hline
\end{tabular}

Tableau 5 : Facteurs associés à la mauvaise qualité de la GATPA en analyse multi variée

\begin{tabular}{|c|c|c|c|c|c|c|}
\hline \multirow[t]{2}{*}{ Variables } & \multirow[t]{2}{*}{$\begin{array}{l}\text { Effectif total } \\
\quad(n=174)\end{array}$} & \multicolumn{2}{|c|}{$\begin{array}{c}\text { Mauvaise qualité } \\
\text { GATPA }\end{array}$} & \multirow[t]{2}{*}{$\begin{array}{c}\text { OR } \\
\text { ajusté }\end{array}$} & \multirow[t]{2}{*}{ IC $95 \%$} & \multirow[t]{2}{*}{$P$ - value } \\
\hline & & Effectif & $\%$ & & & \\
\hline Qualification & & & & & & 0.019 \\
\hline Qualifié & 142 & 82 & 57.7 & 1 & & \\
\hline Non qualifié & 32 & 28 & 87.5 & 3.91 & {$[1.26-12.16]$} & \\
\hline $\begin{array}{l}\text { Relations soignants- } \\
\text { soignés }\end{array}$ & & & & & & $<0.001$ \\
\hline Bonne & 126 & 68 & 54.0 & 1 & & \\
\hline Mauvaise & 48 & 42 & 87.5 & 5.07 & [1.98 - 12.97] & \\
\hline
\end{tabular}




\section{DISCUSSION}

La présente étude a porté sur les facteurs associés à la mauvaise qualité de la GATPA dans sept maternités du district sanitaire de DAGLA en 2011. Cent soixante quatorze (174) accouchements ayant bénéficié de la GATPA ont été analysés; cette taille d'échantillon a été jugée suffisante et a permis de faire des analyses statistiques plus approfondies pour identifier les facteurs associés à la mauvaise qualité de la GATPA. La technique utilisée pour apprécier la qualité de la GATPA a été essentiellement l'observation directe, une des meilleures techniques de collecte de données. Toutefois, la présence des enquêteurs dans les salles d'accouchement pouvait influencer les pratiques des prestataires et entraîner des biais d'observation. Ce biais a été minimisé en recrutant au sein des enquêteurs des infirmières internes moins expérimentées et moins qualifiées ne pouvant intervenir sur la pratique des prestataires plus qualifiés.

\section{Mesure de la qualité de la GATPA dans le district sanitaire de DAGLA}

La bonne qualité de la GATPA dans le district sanitaire de DAGLA a été observée pour $36.8 \%$ des accouchements. Stanton et al. (2009) ont trouvé des proportions faibles de bonne qualité de la GATPA de $0.5 \%$ à $31.8 \%$ dans une étude multicentrique réalisée dans sept pays en 2006 : Bénin, Ethiopie, Tanzanie, Indonésie, El Salvador, Honduras et Nicaragua. En revanche, une meilleure qualité de la GATPA a été observée dans 40,4\%, dans des pays d'Amérique Latine (Vivio et al., 2010). La mauvaise qualité de la GATPA a été moins observée dans notre étude que dans celle réalisée au Bénin en 2006 (63.2\% versus $83.4 \%$ ). Toutefois, ce sont environ deux tiers des accouchements qui n'ont pas bénéficié d'une GATPA correcte; cette mauvaise performance demeure inquiétante, au regard des efforts fournis et ressources déployées par l'Etat et les partenaires techniques et financiers. Les insuffisances ont été constatées aux niveaux du délai d'administration de l'ocytocine, de la contre pression suspubienne, du massage utérin relayé par la femme et du contrôle du tonus. Le délai d'injection de l'ocytocique, au-delà de la minute qui suit la sortie de l'enfant, était dû non pas à sa disponibilité, mais au retard dans les gestes médicaux des prestataires. Ce délai supérieur à la norme recommandée a créé un goulot d'étranglement au niveau de la première composante de la GATPA dont le niveau d'exécution était de $75.3 \%$. Ce résultat est toutefois meilleur à celui de l'enquête nationale de 2006 qui avait trouvé $61.2 \%$ pour cette composante (Stanton et al., 2009). La deuxième insuffisance a été la contre pression sus pubienne qui s'applique simultanément avec la traction contrôlée sur le cordon; cette insuffisance a créé un goulot d'étranglement au niveau de la deuxième composante de la GATPA, avec un niveau d'exécution de $65.5 \%$ versus $45.2 \%$ trouvé dans l'enquête nationale citée ci-dessus. Les troisième et quatrième insuffisances ont été observées au niveau $\mathrm{du}$ «massage relayé par la parturiente » et «le contrôle du tonus utérin » toutes les 15 minutes pendant deux heures. Ces deux insuffisances ont entraîné un goulot d'étranglement au niveau de la troisième composante, avec un niveau d'exécution de $51.1 \%$ contre $17.6 \%$ trouvé dans l'enquête nationale.

\section{Des facteurs associés à la mauvaise qualité de la GATPA dans le district sanitaire de DAGLA}

$\begin{array}{lcc}\text { Des facteurs liés aux ressources } \\ \text { humaines, } & \text { aux } & \text { caractéristiques } \\ \text { socioculturelles et aux antécédents des }\end{array}$


parturientes, au nombre de douze, ont fait l'objet d'analyses bi-variées et multi-variées ; trois facteurs sur quatre liés aux ressources humaines, ont été associés à la mauvaise qualité de la GATPA en analyse bi-variée et deux en analyse multi-variée ; en revanche, un seul facteur lié aux caractéristiques socioculturelles a été associé à la mauvaise qualité de la GATPA en analyse bi-variée et aucun facteur en analyse multi-variée.

Les ressources humaines ont été à la base de la mauvaise application de la GATPA dans la présente étude sur plusieurs plans. Les effectifs des prestataires étaient insuffisants ; en effet, dans les maternités de l'étude, la garde était généralement assurée par deux prestataires au maximum; dans ces conditions, s'il y a deux ou trois accouchements au même moment, le délai d'administration de l'ocytocine peut être supérieur à une minute recommandée et la surveillance des parturientes peut être insuffisante. La qualification et la formation des prestataires permettent une bonne application du protocole clinique; dans la présente étude, la non qualification a été associée à la mauvaise qualité de GATPA. Ce constat est une évidence scientifique qui a été illustrée par Touré et al. (2008) au Mali, qui ont trouvé que l'application de la GATPA était meilleure avec les sages femmes et les infirmières qu'avec les aides soignantes. Ils ont également montré que la GATPA pourrait être pratiquée par les matrones avec un taux de réussite de $96 \%$ obtenu lors de l'évaluation de compétences immédiatement après la formation. Toutefois, la performance théorique est parfois différente de la pratique de routine sur le terrain (performance réelle), surtout si le suivi n'est pas envisagé. Au début de la mise en œuvre de la GATPA au Bénin, seuls les prestataires qualifiés étaient concernés. Mais dans le contexte d'insuffisance de ressources humaines en Afrique au sud du Sahara en général où les aides soignantes et les matrones font plus de $50.0 \%$ des accouchements (Jaffré et Olivier de Sardan, 2003), la pratique de la GATPA a été élargie aux prestataires non qualifiés au Bénin. Cette solution alternative mériterait d'être soutenue par une régularité des supervisions pour renforcer la pratique de la GATPA réalisée par les prestataires non qualifiés. La supervision et la motivation constituent des activités de soutien pour le maintien de la performance. Egalement, les relations prestataires-patients, notamment la communication interpersonnelle, viennent renforcer cette performance. Le personnel non supervisé ou non motivé, qu'il soit qualifié ou pas, appliquera insuffisamment la GATPA. Dans la présente étude, les relations interpersonnelles insuffisantes ont été très significativement associées à la mauvaise qualité de la GATPA. Fourn et al. (2005), dans une étude menée sur la qualité des soins, ont trouvé que la relation soignant-soigné était à la base de l'altération de la qualité des soins et services dans les formations sanitaires. En effet, pour obtenir « le massage relayé par la parturiente » dans la troisième composante de la GATPA, il faut une communication efficace avec celle-ci pour expliquer de façon éclairée l'importance du geste et sa réalisation. L'association des facteurs tels que l'âge, la gestité, la parité, le niveau d'instruction, les grossesses multiples et les interventions gynéco-obstétricales a été également analysée; mais aucune association significative n'a été mise en évidence entre ces variables et la mauvaise qualité de la GATPA. Toutefois, les multi pares peuvent avoir plus d'expérience à appliquer «le massage utérin »; aussi les femmes scolarisées auront plus de facilité à comprendre l'intérêt du massage. Le problème 
de la gestion des utérotoniques relevé dans la présente étude a déjà été décrit par Anglade et al. (2006) dans une étude évaluative sur la politique et les procédures d'utilisation des ocytociques pour l'application de la GATPA au Bénin, au Burkina Faso, au Cameroun et au Mali ; ils ont montré que des ruptures de stock ont été observées en raison des facteurs, tels que l'absence d'estimation des stocks, le système d'approvisionnement inefficace et l'utilisation irrationnel $\mathrm{du}$ produit. $\mathrm{La}$ préparation ainsi que l'application proprement dite de la GATPA méritent également une attention particulière ; au cours de ces différentes étapes, certaines complications peuvent survenir, telles que la déchirure des membranes placentaires, le mauvais décollement $\mathrm{du}$ placenta, la rupture prématurée du cordon. La prise en charge de ces complications nécessite la présence des agents qualifiés capables d'intervenir immédiatement. La présente étude a retenu dans le modèle final l'importance de la qualification des prestataires et les relations interpersonnelles prestataires-patients comme facteurs influençant la qualité de la GATPA. Ces résultats viennent une fois encore soulever la problématique de l'utilisation des personnels non qualifiés, les aides soignantes et les matrones, dans la réalisation de certains actes médicaux, tels que la consultation prénatale et l'accouchement (Harvey et al., 2007). La formation continue en cours d'emploi, présente des limites sur le plan pédagogique et de son appropriation effective par les bénéficiaires ; c'est pour ces raisons que la GATPA devra être intégrée dans les curricula de formation initiale des agents de santé qualifiés. Enfin, les défis pour la mise en œuvre efficace de la GATPA intéressent les six domaines de renforcement du système de santé retenus par l'OMS à savoir: les prestations de service, le personnel de santé, les informations, les produits médicaux et technologiques, le financement, le leadership et la gouvernance (OMS, 2007). Trois domaines ont été abordés et partiellement dans la présente étude : le personnel de santé, les prestations de services, les médicaments et matériels médicaux. En plus de l'approfondissement de ces domaines par des études, les autres méritent d'être investigués.

\section{Conclusion}

La présente étude menée dans le district sanitaire de DAGLA a montré que la mauvaise qualité de la GATPA a été de $63.2 \%$ et constitue un problème de prise en charge obstétricale. L'étude a mis en exergue les ressources humaines comme facteur responsable de cette mauvaise qualité. Les personnels de santé qualifiés constituent la composante principale de la mise en œuvre des interventions à haut impact dont la GATPA et devront être valorisés à travers la formation, la motivation, les conditions de travail et la supervision. Toutefois, les obstacles à la mise en œuvre de la GATPA ne se résument pas aux seules ressources humaines ; les autres composantes de renforcement du système de santé devront être prises en compte ainsi que l'intégration de la GATPA dans les soins courants à chaque accouchement.

\section{REFERENCES}

Abalos E. 2009. Prise en Charge Active contre Expectative au Cours de la Délivrance : Commentaire de la BSG. Bibliothèque de Santé Génésique de l'OMS : Genève.

Anglade MK. 2006. Revue de la politique et des procédures d'utilisation des ocytociques pour la gestion active de la troisième phase de l'accouchement et la prévention d'hémorragie du post-partum 
dans quatre pays africains : Bénin, Burkina Faso, Cameroun et Mali. Rational Pharmaceutical Management Plus Program. MSH / USAID, Arlington/USA.

Bishop RA, Litch JA, Tuggy M. 2008. Active Management of the Third Stage of Labor (AMTSL): A Clinical Tutorial. Seattle: PATH.

Burke C. 2010. Active versus expectant management of the third stage of labor and implementation of a protocol. $J$. Perinat. Neonatal Nurs., 24(3): 215-228.

Cynthia S, Deborah A, Rod K. 2009. Utilisation de la gestion active de la troisième phase du travail dans sept pays en développement. Bull World Health Organization, 87: 207-215.

DDS-ZC du Bénin. 2010. (Direction Départementale Santé du Zou-Collines du Bénin). Evaluation de la qualité de la gestion des services de santé 2. PISAF/ USAID \& DDS-ZC, Bohicon.

Fourn L, Ouendo EM, Adé G, Kassa F. 2005. Qualité des soins : Approche centrée sur le malade. Médecine d'Afrique Noire, 52(2): 91-95.

Harvey SA, Blandón CW, McCaw-Binns A, Sandino I, Urbina L, Rodriguez C, Gómez I, Ayabaca P, Djibrina S, the Nicaraguan Maternal and Neonatal Health Quality Improvement Group. 2007. Les «accoucheurs/euses qualifiés/es» ont-ils/elles réellement les compétences pour pratiquer des accouchements? Présentation d'une méthode de mesure de ces compétences, de certains résultats dérangeants et d'une voie de progression potentielle. Bulletin de l'Organisation Mondiale de la Santé, 85(10): 783-790.

Jaffré Y, Olivier de Sardan JP. 2003. Une Médecine Inhospitalière. Les Difficiles
Relations entre Soignants et Soignés dans cinq Capitales d'Afrique de l'Ouest. Karthala: Paris.

Khan KS, Wojdyla D, Say L, Gulmezoglu AM, Van Look PFA. 2006. OMS analysis of causes of maternal mortality: a systematic review. Lancet, 367(9516): 1066-1074.

Lawn JE, Darmstadt GL, Organizing Guest Editors et RL Goldenberg, External Guest Editor. 2009. Intrapartum-related deaths: evidence for Action. International Journal of Gynecology \& Obstetrics, 107(Supplement 1): 9-150.

Mathai M, Gulmezoglu AM, Hill S. 2007. WHO Recommendations for the Prevention of Postpartum Hemorrhage. OMS: Genève.

Ministère de la Santé Publique du Bénin. 2009. Protocoles des Services de Santé Familiale: Volet Femme (Deuxième Edition). DSF en collaboration avec UNFPA \& USAID : Cotonou.

Organisation Mondiale de la Santé. 2007. Everybody's business: Strengthening health systems to improve health outcomes: WHO's framework for action. OMS, Genève.

PPHI (Prevention of PostPartum Hemorrhage Initiative). 2008. La prévention de l'hémorragie du post-partum : la gestion active de la troisième période de l'accouchement. Formation d'accoucheurs qualifiés. Manuel de Référence, Seattle, PATH.

Prendiville WJP, Elbourne D, McDonald SJ. 2000. Active Versus Expectant Management in the Third Stage of Labour. The Cochrane Library: Chichester.

Stanton C, Armbruster D, Knight R, Ariawan I, Gbangbade S, Getachew A, Portillo JA, Jarquin D, Marin F, Mfinanga S, 
Vallecillo J, Johnson H, Sintasath D. 2009. Application de la prise en charge active du troisième stade du travail dans sept pays en développement. Bulletin de l'Organisation Mondiale de la Santé, 87 (3): 207-215.

Touré CO, Kéita B, Susheela E, Konaté R. 2008. Evaluation finale sur la Faisabilité de la Gestion Active de la Troisième Période de l'Accouchement par les Matrones au Mali. DSR/ MS : Bamako.

Vivio D, Fullerton JT, Forman R, Mbewe RK, Musumali M, Chewe PM. 2010. Integration of the practice of active management of the third stage of labor within training and service implementation programming in Zambia. J. Midwifery Women's Health, 55(5): 447-54. 